\title{
Unveiling the nature of the unidentified gamma-ray sources: blazar counterparts at low radio frequencies
}

\author{
Francesco Massaro, R. D'Abrusco, M. Giroletti, A. Paggi, \\ N. Masetti and G. Tosti \\ Stanford University, CA, USA \\ E-mail: fmassaro@stanford.edu
}

\begin{abstract}
About one third of the gamma-ray sources detected by Fermi have still no firmly established counterpart at lower energies. Here we propose a new approach to find candidate counterparts for the unidentified gamma-ray sources (UGSs) based on the $325 \mathrm{MHz}$ radio survey performed with Westerbork Synthesis Radio Telescope (WSRT) in the northern hemisphere. First we investigate the low-frequency radio properties of blazars, the largest known population of gamma-ray sources; then we search for sources with similar radio properties combining the information derived from the Westerbork Northern Sky Survey (WENSS) with those of the NRAO VLA Sky survey (NVSS). We present a list of candidate counterparts for 32 UGSs with at least one counterpart in the WENSS. We also performed an extensive research in literature to look for infrared and optical counterparts of the gamma-ray blazar candidates selected with the low-frequency radio observations to confirm their nature. On the basis of our multifrequency research we identify 23 new gamma-ray blazar candidates out of 32 UGSs investigated. I will also present the first analysis of very low frequency radio emission of blazars based on the recent Very Large Array Low-Frequency Sky Survey (VLSS) at $74 \mathrm{MHz}$. I show that blazars present radio flat spectra when evaluated at $74 \mathrm{MHz}$, about an order of magnitude in frequency lower than previous analyses. The implications of these findings in the contest of the blazars - radio galaxies connection will be discussed.
\end{abstract}

